\title{
Low circulating microRNA levels in heart failure patients are associated with atherosclerotic disease and cardiovascular-related rehospitalizations
}

\author{
Eline L. Vegter ${ }^{1} \cdot$ Ekaterina S. Ovchinnikova $^{1,2} \cdot$ Dirk J. van Veldhuisen ${ }^{1}$ • \\ Tiny Jaarsma ${ }^{3} \cdot$ Eugene Berezikov $^{2} \cdot$ Peter van der Meer $^{1} \cdot$ Adriaan A. Voors $^{1}$
}

Received: 29 September 2016 / Accepted: 22 February 2017 / Published online: 14 March 2017

(c) The Author(s) 2017. This article is published with open access at Springerlink.com

\begin{abstract}
Objective Circulating microRNAs (miRNAs) have been implicated in both heart failure and atherosclerotic disease. The aim of this study was to examine associations between heart failure specific circulating miRNAs, atherosclerotic disease and cardiovascular-related outcome in patients with heart failure.

Methods The levels of 11 heart failure-specific circulating miRNAs were compared in plasma of 114 heart failure patients with and without different manifestations of atherosclerotic disease. We then studied these miRNAs in relation to biomarkers associated to atherosclerosis and to cardiovascular-related rehospitalizations during 18 months of follow-up.

Results At least one manifestation of atherosclerotic disease was found in $70(61 \%)$ of the heart failure patients. A consistent trend was found between an increasing number of manifestations of atherosclerosis (peripheral arterial disease in specific), and lower levels of miR-18a-5p, miR27a-3p, miR-199a-3p, miR-223-3p and miR-652-3p (all
\end{abstract}

Electronic supplementary material The online version of this article (doi:10.1007/s00392-017-1096-z) contains supplementary material, which is available to authorized users.

Adriaan A. Voors

a.a.voors@umcg.nl

1 Department of Cardiology, AB31, University Medical Center Groningen, Hanzeplein 1, 9713 GZ Groningen, The Netherlands

2 European Research Institute for the Biology of Ageing, University of Groningen, Antonius Deusinglaan 1, 9713 AV Groningen, The Netherlands

3 Faculty of Medical and Health Sciences, University of Linkoping, 58183 Linköping, Sweden
$P<0.05)$. Target prediction and network analyses identified several interactions between miRNA targets and biomarkers related to inflammation, angiogenesis and endothelial dysfunction. Lower miRNA levels were associated with higher levels of these atherosclerosis-related biomarkers. In addition, lower miRNA levels were significantly associated with rehospitalizations due to cardiovascular causes within 18 months, with let-7i-5p as strongest predictor [HR 2.06 (95\% CI 1.29-3.28), C-index 0.70, $P=0.002$ ].

Conclusions A consistent pattern of lower levels of circulating miRNAs was found in heart failure patients with atherosclerotic disease, in particular peripheral arterial disease. In addition, lower levels of miRNAs were associated with higher levels of biomarkers involved in atherosclerosis and an increased risk of a cardiovascular-related rehospitalization.

Keywords Circulating microRNAs $\cdot$ Heart failure · Atherosclerosis $\cdot$ Biomarkers $\cdot$ Rehospitalization

\section{Introduction}

MicroRNAs (miRNAs) can function as important regulators of a wide range of biological processes and contribute to the development of various diseases, including heart failure [1]. These small, non-coding RNAs are potent regulators of gene expression, which function via binding to the target messenger RNA (mRNA). This in turn leads to either degradation of the mRNA or to repression of translation, resulting in a disturbed protein synthesis [2]. Extracellular miRNAs can be detected in circulating blood, and have shown to function as potential biological markers in heart failure [1, 3, 4]. A variety of studies identified differentially expressed circulating miRNAs in heart failure [5-7]. We 
recently reported about a panel of circulating miRNAs that consistently showed lower plasma levels in (acute) heart failure patients compared to healthy controls [7]. A gradual increase in miRNA levels was seen towards more stabilized heart failure patients, chronic heart failure patients and healthy controls.

Remarkably, no cardiac specific or cardiac-enriched miRNAs (such as miR-1, miR-133, miR-499 and miR-208) were present in this set of heart failure-related miRNAs, suggesting that the most differentially expressed miRNAs in the circulation of heart failure patients do not originate from the heart. Several studies showed that blood and endothelial cells are the major source of abundant miRNAs in the circulation $[8,9]$ and literature on our previously found miRNA signature in heart failure revealed potential involvement in vascular-related processes including angiogenesis, endothelial dysfunction and inflammation [10-15]. Disturbances in these processes are frequently present in patients with atherosclerosis [16], therefore we hypothesized that the previously found circulating miRNAs in heart failure might be related to atherosclerosis and underlying vascular disease processes.

To investigate this, we measured the previously established heart failure-related miRNA panel in another cohort of 114 heart failure patients, consisting of patients with and without atherosclerotic disease. We aimed to identify differences in circulating heart failure-related miRNA levels in heart failure patients with and without different clinical manifestations of atherosclerosis, including coronary artery disease (CAD), a medical history of stroke or transient ischemic attack (TIA) and peripheral arterial disease (PAD). In addition, we studied associations between miRNA levels and biomarkers related to atherosclerotic disease processes such as inflammation, angiogenesis and endothelial dysfunction, and we assessed the relation with the risk of rehospitalization due to cardiovascular (CV)related causes.

\section{Materials and methods}

\section{Study population}

From the 1023 patients of the Coordinating Study Evaluating Outcomes of Advising and Counseling in Heart Failure $(\mathrm{COACH})$, a subset of 114 randomly selected patients was studied based on the availability of plasma samples and complete biomarker measurements at baseline. The main results of the $\mathrm{COACH}$ study were previously published [17]. Briefly, the COACH study investigated the effect of additional specialized nurse-led support with different intensities on outcome parameters in patients with heart failure. All patients had been admitted to the hospital with symptoms of heart failure, New York Heart Association (NYHA) functional classification II to IV. Blood samples were collected shortly before discharge. Data on disease history was collected from the medical charts. Patients were divided according to the presence of $0,1,2$ or 3 manifestations of atherosclerotic disease. The three manifestations of atherosclerotic disease consisted of CAD (defined as either a medical history of a myocardial infarction and/ or a revascularization procedure by means of percutaneous coronary intervention (PCI) or coronary artery bypass grafting (CABG) surgery), TIA or stroke (defined as a medical history of either a TIA and/or a stroke) and PAD (defined as a medical history of PAD). Healthy control subjects $(n=10)$ were derived from the Telosophy study [18]. Main exclusion criteria of the control subjects were presence of heart failure, a family history of premature $\mathrm{CV}$ disease and known atherosclerotic disease.

\section{MicroRNA measurements}

RNA was isolated from plasma samples using the miRCURY RNA isolation kit for bodyfluids from Exiqon (Vedbaek, Denmark). The Universal cDNA Synthesis kit (Exiqon) was used for the reversed transcription reactions. The levels of the following previous identified circulating miRNAs [7] in heart failure patients were determined in plasma from 114 heart failure patients using a customized Exiqon miRNA PCR panel; let-7i-5p, miR-16-5p, miR-18a-5p, miR-26b-5p, miR-27a-3p, miR-30e-5p, miR106a-5p, miR-199a-3p, miR-223-3p, miR-423-5p and miR-652-3p. Circulating levels of these miRNAs were also measured in plasma samples from ten healthy control subjects, as previously described [7]. Polymerase chain reactions were performed on the LightCycler $^{\circledR} 480$ (Roche Applied Science, Rotkreuz, Switzerland) with cycle settings as recommended by Exiqon. Synthetic RNA templates were used to control for isolation yield (UniSp4), cDNA synthesis (UniSp6) and PCR efficiency (UniSp3). Only miRNAs with $\mathrm{Ct}$ values less than 37 were included in the further analyses. The miRNA let-7a-5p was selected as best performing reference gene in the investigated cohorts, as determined by GeNorm and NormFinder (GenEx Professional software, MultiD Analyses, Sweden). Expression levels of the measured miRNAs were normalized against miRNA let-7a-5p using the GenEx Professional software and the delta $\mathrm{Ct}$ method was performed to obtain the relative miRNA expression levels (the $\mathrm{Ct}$ value of the reference miRNA was subtracted from the $\mathrm{Ct}$ value of the target miRNA). High miRNA expression is reflected by low delta $\mathrm{Ct}$ values (representing a low number of fractional cycles needed to reach the threshold of the amplified target miRNA), and low miRNA expression by high delta $\mathrm{Ct}$ values. 


\section{Biomarker measurements}

Plasma concentrations of the majority of atherosclerosisrelated biomarkers were measured by Alere ${ }^{\mathrm{TM}}$, San Diego, CA. Competitive ELISAs on a Luminex ${ }^{\circledR}$ platform were used to measure the biomarkers angiogenin, C-reactive protein (CRP), D-dimer, endothelial cell-selective adhesion molecule (ESAM), growth differentiation factor 15 (GDF15), lymphotoxin beta receptor (LTBR), myeloperoxidase (MPO), neutrophil gelatinase-associated lipocalin (NGAL), neuropilin-1, osteopontin, pentraxin-3, polymeric immunoglobulin receptor (PIGR), receptor for advanced glycation endproducts (RAGE), syndecan-1, tumor necrosis factor alpha receptor 1 (TNFR-1), tumor necrosis factor receptor superfamily member (troy) and vascular endothelial growth receptor 1 (VEGFR-1). Endothelin-1 and interleukin-6 (IL-6) were measured by means of the high sensitive single molecule counting (SMC ${ }^{\mathrm{TM}}$ ) technology (RUO, Erenna ${ }^{\circledR}$ Immunoassay System) by Singulex Inc. (Alameda, CA, USA). Galectin-3 was measured using the BG Medicine galectin-3 assay (BG Medicine, Waltham, MA), more extensively described elsewhere [19]. The inter- and intraassay coefficients of variation for each of the biomarkers were previously published [20].

\section{Target prediction and network analysis}

Potential targets of the set of circulating miRNAs were predicted using miRTarBase 6.0 [21]. Only experimentally validated targets (by means of reporter assay, western blot, microarray or next-generation sequencing) were selected to increase the reliability of the identified targets. Next, an interaction network of the overlapping miRNA targets (i.e. genes targeted by more than one of the investigated miRNAs) was created using STRING v.10 [22].

\section{Statistical analyses}

GenEx Professional software (MultiD Analyses, Sweden) was used for the raw miRNA expression data. Other statistical analyses were conducted with R: A Language and Environment for Statistical Computing, version 3.2.0 ( $\mathrm{R}$ Foundation for Statistical Computing, Vienna, Austria). Normally distributed variables were depicted as mean \pm standard deviation and non-normally distributed variables were presented as median with the interquartile range. Differences between groups were determined using $\mathrm{t}$ tests for normally distributed continuous variables and Mann-Whitney $U$ tests for non-normally distributed continuous variables. For binomially and categorical variables, the Chi-square test was used. Linear trend tests were used for miRNA and biomarker levels across groups and quartiles. To examine the predictive value of miRNAs for various endpoints uni- and multivariable Cox proportional hazards regression analyses were performed. $P$ values of $<0.05$ were considered significant.

\section{Results}

\section{Baseline characteristics of the study population}

Baseline characteristics of the 114 hospitalized heart failure patients at time of discharge are presented in Supplementary Table 1 . Patient characteristics were similar to the complete $\mathrm{COACH}$ population, mostly male, with a mean age of $71.1( \pm 10.4)$ years and median NT-proBNP of 3566 [1661-7848] pg/mL. Forty-four patients (39\%) had no atherosclerotic disease and $70(61 \%)$ had at least one manifestation of atherosclerotic disease. From the 114 patients, $54 \%$ showed evidence of CAD, $13 \%$ had a medical history of a previous stroke and/or TIA and $21 \%$ had PAD.

\section{Circulating microRNA levels in patients with heart failure compared to controls}

To confirm our previous findings of lower miRNA levels in heart failure patients compared to control subjects, we compared the circulating miRNA levels of the 114 patients to a control cohort consisting of ten healthy subjects. Baseline characteristics of the control population are depicted in Supplementary Table 1. In concordance with our previous study [7], we found lower levels of the majority of the heart failure-related circulating miRNAs in heart failure patients compared to controls, with the exception of miR423-5p and miR-16-5p showing higher and unchanged levels, respectively (Supplementary Fig. 1). Statistically significant lower levels in heart failure patients compared to healthy individuals were found for miR-18a-5p, miR26b-5p, miR-27a-3p, miR-30e-5p, miR-199a-3p and miR223-3p (Table 1).

\section{Associations of microRNA levels with the number of different manifestations of atherosclerosis}

Next, we assessed the relation between the extensiveness of atherosclerotic disease in heart failure patients and circulating miRNA levels. Patients were divided based on the number of different manifestations of atherosclerosis, including the presence of CAD, PAD and a history of stroke/TIA. For the majority of the miRNAs, the same pattern could be observed in which miRNA levels decreased in parallel with an increase of different manifestations of atherosclerotic disease (Table 2). The gradual decline in plasma levels of miR-18a-5p, miR-27a-3p, miR-199a-3p, miR-223-3p and miR-652-3p were statistically significant. 
Table 1 Circulating microRNA levels in heart failure patients (HF) compared to control subjects

\begin{tabular}{llcc}
\hline Variable & Controls & HF & $P$ value \\
$N$ & 10 & 114 & \\
\hline let-7i-5p & $0.5 \pm 0.5$ & $0.8 \pm 1$ & 0.095 \\
miR-16-5p & $-6.1 \pm 1.1$ & $-6 \pm 1.3$ & 0.819 \\
miR-18a-5p & $1.1 \pm 0.6$ & $2.5 \pm 1.1$ & $<0.001$ \\
miR-26b-5p & $2.1 \pm 0.6$ & $3.7 \pm 0.9$ & $<0.001$ \\
miR-27a-3p & $-1.8 \pm 0.6$ & $-0.5 \pm 1.1$ & $<0.001$ \\
miR-30e-5p & $-0.6 \pm 0.6$ & $0 \pm 1.2$ & 0.028 \\
miR-106a-5p & $-1.7[-1.9$ to 1.3$]$ & $-0.6 \pm 1$ & 0.079 \\
miR-199a-3p & $-0.6 \pm 0.6$ & $0.6 \pm 1$ & $<0.001$ \\
miR-223-3p & $-5.2[-5.5$ to 4.8$]$ & $-4.5 \pm 1.2$ & 0.002 \\
miR-423-5p & $0.5 \pm 1$ & $-0.3 \pm 1$ & 0.028 \\
miR-652-3p & $0.8 \pm 0.5$ & $1.3 \pm 1$ & 0.060 \\
\hline
\end{tabular}

Values represent the normalized (delta $\mathrm{Ct}$ ) miRNA levels presented as mean \pm standard deviation or median with interquartile range (in square brackets)

\section{Differences in circulating microRNA levels in heart failure patients with coronary artery disease, a medical history of stroke or transient ischemic attack, and peripheral arterial disease}

To examine the effects of different manifestations of atherosclerotic disease on miRNA levels in more detail, we determined the differences in miRNA levels between the heart failure patients with or without CAD, a medical history of TIA/stroke and PAD. Clinical characteristics of patients belonging to these different categories of atherosclerosis are depicted in Table 3. There were no consistent trends in plasma levels of the selected miRNAs in patients with and without CAD (Supplementary Table 2A) and patients with a previous stroke or TIA (Supplementary Table 2B). In heart failure patients with PAD, several miRNA differences were found compared to heart failure patients without PAD. Plasma concentrations of all miRNAs (except for miR-423-5p) were lower in heart failure patients with PAD compared to heart failure patients without PAD (Table 4) and miR-18a-5p, miR-27a-3p, miR-30e-5p, miR-106a-5p, miR-199a-3p, miR-223-3p and miR-652-3p showed significantly lower circulating miRNA levels. Notably, heart failure patients with PAD had lower diastolic blood pressure and more patients had CAD (Table 3). Further, patients with PAD more often developed renal impairment with higher creatinine and potassium levels and a lower estimated glomerular filtration rate. The majority of the associations between the differentially expressed miRNAs and the presence of PAD remained after adjustment for these variables.

\section{Associations between circulating microRNAs and biomarkers}

We performed target prediction analysis to identify the experimentally validated potential targets of the panel of heart failure-related miRNAs. We selected the overlapping targets (i.e. mRNAs targeted by more than one of the investigated miRNAs) and show that the majority of these targets interact which each other, as presented in the network figure (Supplementary Fig. 2). Genes with a central

Table 2 Circulating microRNA levels in patients with $0,1,2$ or 3 different manifestations of atherosclerotic disease

\begin{tabular}{lcccc}
\hline Variable & 0 & 1 & 2 & 3 \\
& & CAD $n=37$ & CAD $n=21$ & CAD $n=4$ \\
& & PAD $n=3$ & PAD $n=17$ & PAD $n=4$ \\
& & Stroke/TIA $n=3$ & Stroke/TIA $n=8$ & Stroke/TIA $n=4$ \\
$N$ & 44 & 43 & 23 & 4 \\
\hline let-7i-5p & $0.9 \pm 0.8$ & $0.7 \pm 1$ & $0.8 \pm 1.1$ & $0.6 \pm 1.1$ \\
miR-16-5p & $-6 \pm 1.1$ & $-6.1 \pm 1.4$ & $-6 \pm 1.4$ & $-5.9 \pm 1.7$ \\
miR-18a-5p & $2.3 \pm 0.9$ & $2.5 \pm 1.3$ & $2.8 \pm 1.1$ & $3.6 \pm 1.5$ \\
miR-26b-5p & $3.8 \pm 0.9$ & $3.6 \pm 0.9$ & $3.8 \pm 1.1$ & $3.9 \pm 0.6$ \\
miR-27a-3p & $-0.7 \pm 0.9$ & $-0.5 \pm 1.3$ & $-0.5 \pm 0.9$ & $0.7 \pm 1.7$ \\
miR-30e-5p & $0 \pm 1.2$ & $-0.2 \pm 1.2$ & $0.2 \pm 1.2$ & $0.7 \pm 1.8$ \\
miR-106a-5p & $-0.8 \pm 0.9$ & $-0.6 \pm 1.1$ & $-0.3 \pm 1.1$ & $0.1 \pm 1.4$ \\
miR-199a-3p & $0.5 \pm 0.9$ & $0.5 \pm 1$ & $0.7 \pm 0.9$ & 0.718 \\
miR-223-3p & $-4.8 \pm 0.9$ & $-4.4 \pm 1.2$ & $-4.1 \pm 1.3$ & 0.014 \\
miR-423-5p & $-0.1 \pm 0.7$ & $-0.4 \pm 1.2$ & $-0.5 \pm 0.9$ & 0.178 \\
miR-652-3p & $1.2 \pm 0.8$ & $1.2 \pm 1.1$ & $1.6 \pm 0.8$ & $-3.5 \pm 2.4$ \\
\hline
\end{tabular}

Number of manifestations of atherosclerotic disease are presented, including coronary artery disease (CAD), peripheral arterial disease (PAD) and stroke/transient ischemic attack (TIA). Values represent the normalized (delta Ct) miRNA levels presented as mean \pm standard deviation 


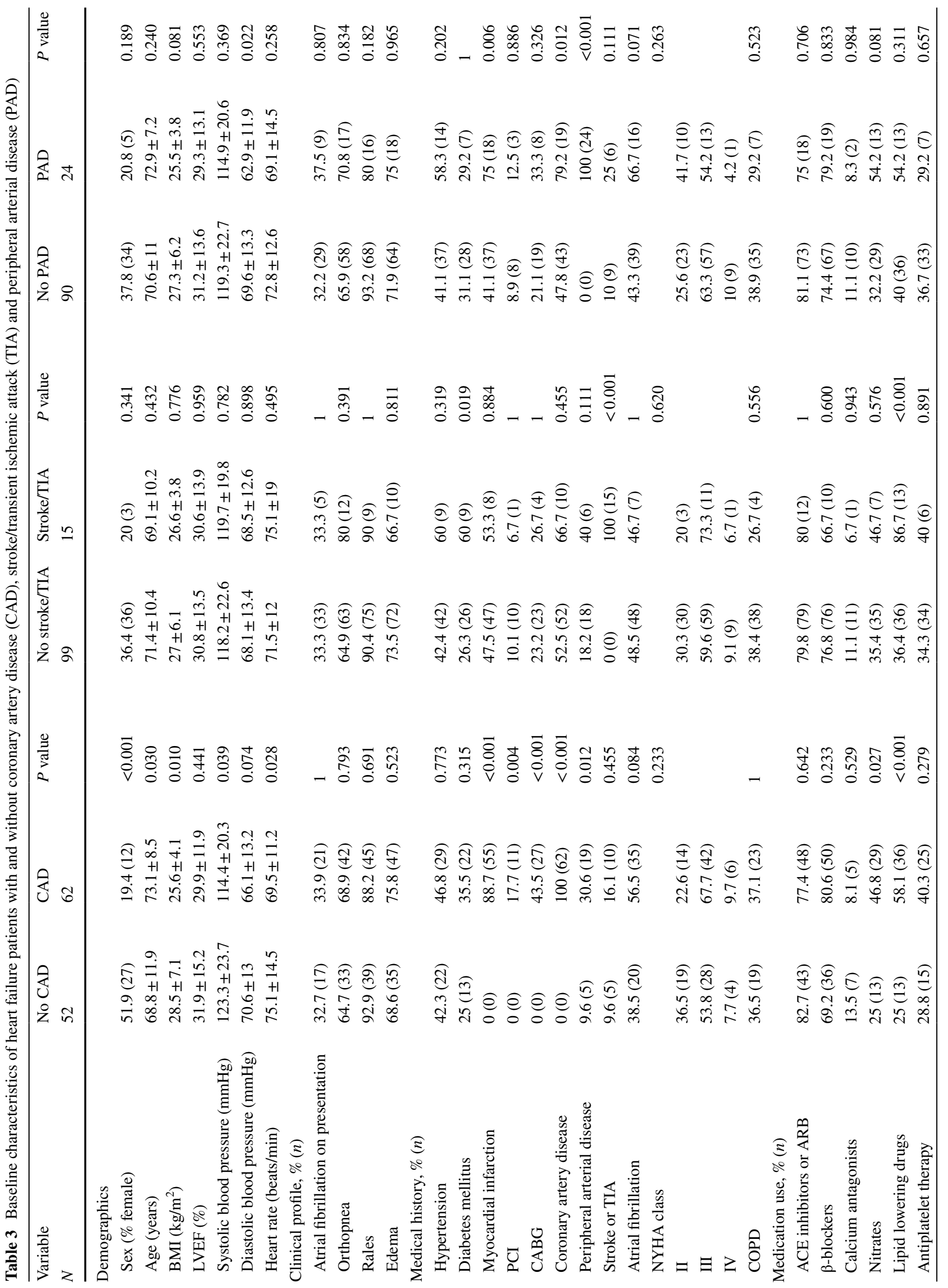




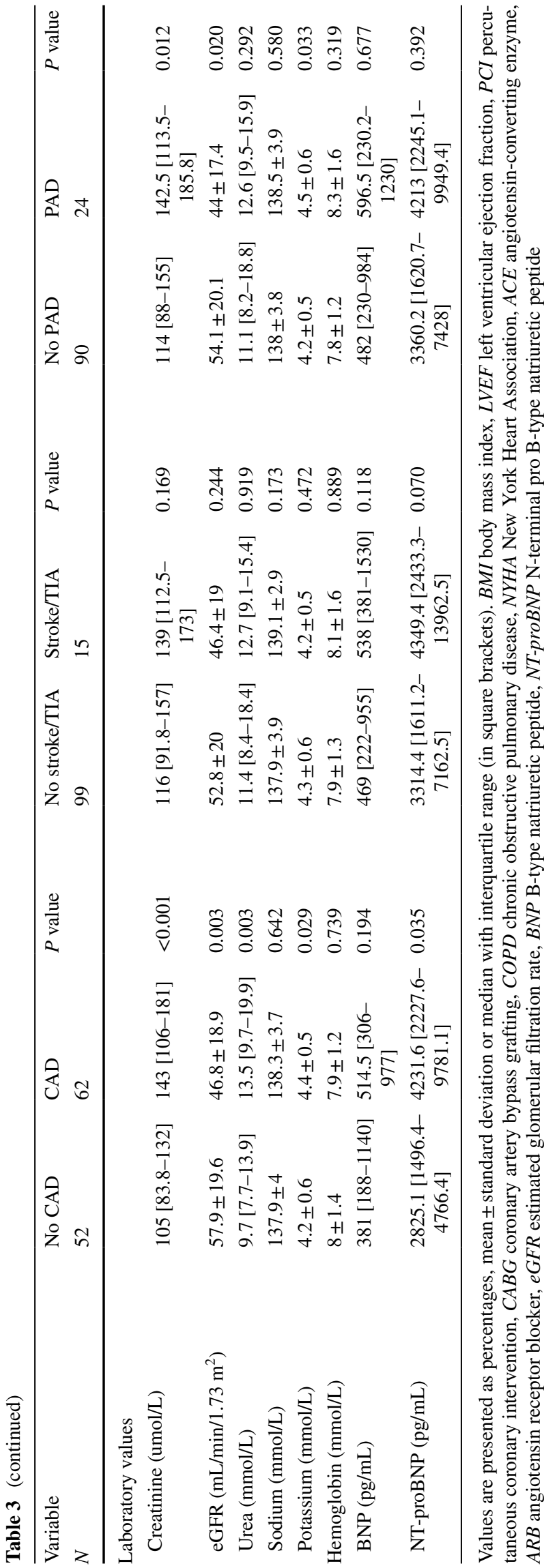

position in the network and multiple interactions with other target genes include FOXO1, MAPK14, CDK2, PTEN and SP1.

Biomarkers were selected based on known associations with atherosclerosis, inflammation, angiogenesis and endothelial dysfunction. We found a total of 201 interactions between the set of biomarkers and all predicted miRNA targets (in total 213), resulting from the network analysis (Supplementary Table 3). MiRNAs differentially expressed in heart failure patients with an increasing number of manifestations of atherosclerosis and PAD were divided in quartiles based on their expression levels after which the trend with biomarker levels was determined (Table 5). A significant P-for-trend was observed for multiple biomarkers showing consistent trends of high levels in the patients with the lowest miRNA levels, including ESAM, LTBR, PIGR, pentraxin-3, troy, syndecan-1, galectin-3, NGAL, GDF-15, RAGE, TNFR-1, neuropilin-1 and angiogenin. Low levels of miR-18a-5p, miR-106a-5p and miR-223-3p were significantly associated with high levels of a variety of mainly inflammatory and endotheliumrelated biomarkers (ESAM, LTBR, PIGR, syndecan-1, GDF-15, RAGE, TNFR-1, pentraxin-3, galectin-3, troy), whereas low levels of miR-27a-3p and miR-199a-3p were related to high levels of biomarkers important in angiogenesis-related processes (galectin-3, neuropilin-1 and angiogenin), as summarized in Fig. 1.

\section{Predictive value of circulating microRNAs and cardiovascular-related rehospitalization}

We studied the association between our set of established circulating miRNAs and CV-related endpoints. Within 18 months, 28 events of rehospitalization resulted from

Table 4 Circulating microRNA levels in heart failure patients with and without peripheral arterial disease (PAD)

\begin{tabular}{lccr}
\hline Variable & No PAD & PAD & $P$ value \\
$N$ & 90 & 24 & \\
\hline let-7i-5p & $0.7 \pm 0.9$ & $1.1 \pm 1$ & 0.112 \\
miR-16-5p & $-6.1 \pm 1.2$ & $-5.8 \pm 1.4$ & 0.248 \\
miR-18a-5p & $2.4 \pm 1.1$ & $3.1 \pm 1$ & 0.006 \\
miR-26b-5p & $3.7 \pm 0.9$ & $4 \pm 0.9$ & 0.118 \\
miR-27a-3p & $-0.7 \pm 1.1$ & $-0.1 \pm 1$ & 0.018 \\
miR-30e-5p & $-0.2 \pm 1.2$ & $0.7 \pm 1.2$ & 0.004 \\
miR-106a-5p & $-0.7 \pm 1$ & $-0.1 \pm 1$ & 0.013 \\
miR-199a-3p & $0.4 \pm 1$ & $1 \pm 0.9$ & 0.010 \\
miR-223-3p & $-4.6 \pm 1.2$ & $-3.8 \pm 1.3$ & 0.005 \\
miR-423-5p & $-0.3 \pm 1$ & $-0.3 \pm 0.9$ & 0.986 \\
miR-652-3p & $1.1 \pm 1$ & $1.9 \pm 0.8$ & $<0.001$ \\
\hline
\end{tabular}

Values represent the normalized (delta $\mathrm{Ct}$ ) miRNA levels presented as mean \pm standard deviation 
Table 5 Circulating microRNAs significantly associated with atherosclerosis-related biomarkers

\begin{tabular}{|c|c|c|c|c|c|}
\hline miRNA quartiles & Q1 & Q2 & Q3 & Q4 & P-for-trend \\
\hline$N$ & 29 & 28 & 28 & 29 & \\
\hline \multicolumn{6}{|l|}{$\operatorname{miR}-18 a-5 p$} \\
\hline ESAM & $56.5 \pm 16.8$ & $60.9 \pm 14.6$ & $64 \pm 21.6$ & $65.4 \pm 16.7$ & 0.048 \\
\hline Galectin-3 & $20.4 \pm 9.5$ & $22.5 \pm 8$ & $25.3 \pm 11$ & $27.1 \pm 10.4$ & 0.007 \\
\hline LTBR & $0.7[0.5-1]$ & $0.8[0.5-1.1]$ & $0.8[0.5-1.4]$ & $0.9[0.7-1.5]$ & 0.017 \\
\hline Pentraxin-3 & $3.8 \pm 2.2$ & $4.1 \pm 1.6$ & $4.8 \pm 2.6$ & $4.8 \pm 2.3$ & 0.045 \\
\hline PIGR & 695.3 [413.4-1081.1] & 617.4 [512.4-998.1] & 774.5 [439.7-1175] & 831.6 [612.6-1194.2] & 0.043 \\
\hline RAGE & $3[2.1-5]$ & $2.7[1.8-4.3]$ & $3.8[2.7-6.1]$ & $5[3.7-6.3]$ & 0.034 \\
\hline Syndecan-1 & $19.7[13.5-25.4]$ & $20.8[16.2-25.5]$ & $24.9[16.3-32.2]$ & $22.4[18.8-28.2]$ & 0.030 \\
\hline TNFR-1 & $3.1[2.1-4.2]$ & $3.4[2.3-4.7]$ & $3.3[2.3-7.2]$ & $4.6[3-6.8]$ & 0.037 \\
\hline Troy & $0.9[0.8-1.5]$ & $1[0.9-1.6]$ & $1.2[0.7-2]$ & $1.5[0.9-2.2]$ & 0.005 \\
\hline \multicolumn{6}{|l|}{ miR-30e-5p } \\
\hline Galectin-3 & $21.5 \pm 7.4$ & $22.6 \pm 9.7$ & $25.2 \pm 11.8$ & $26.3 \pm 10.4$ & 0.043 \\
\hline \multicolumn{6}{|l|}{$\operatorname{miR}-27 a-3 p$} \\
\hline Galectin-3 & $19.9 \pm 7.5$ & $23.5 \pm 7.4$ & $23.2 \pm 10.4$ & $29.1 \pm 12$ & 0.001 \\
\hline Neuropilin-1 & $871.2 \pm 234.6$ & $953.1 \pm 265.8$ & $1007.6 \pm 324.1$ & $1091.5 \pm 333.7$ & 0.004 \\
\hline NGAL & $107.3[84.3-161.3]$ & 135.4 [101.1-169.9] & $151.1[103.4-178.2]$ & $147.7[112.8-229.3]$ & 0.006 \\
\hline \multicolumn{6}{|l|}{ miR-106a-5p } \\
\hline ESAM & $55.9 \pm 17.1$ & $60.7 \pm 17.1$ & $57.4 \pm 14.9$ & $72.7 \pm 16.9$ & 0.001 \\
\hline Galectin-3 & $20.6 \pm 9.1$ & $20.1 \pm 5.8$ & $24.9 \pm 10.9$ & $30.1 \pm 10.3$ & $<0.001$ \\
\hline GDF-15 & $2.7[1.8-4.4]$ & $3[1.9-5.3]$ & $3.3[1.8-6.1]$ & $4.1[3.2-6.4]$ & 0.012 \\
\hline LTBR & $0.6[0.4-0.8]$ & $0.8[0.6-1.2]$ & $0.8[0.5-1.2]$ & $1.2[0.7-1.7]$ & $<0.001$ \\
\hline PIGR & 559 [415.9-1029.5] & 666.4 [421.4-1061.6] & 689.6 [424.5-894.1] & 1024.9 [778.2-1625.8] & $<0.001$ \\
\hline RAGE & $3.1[2.3-4.4]$ & $3.4[2.2-6]$ & $3.4[2.4-5.5]$ & $5[2.9-8.3]$ & 0.011 \\
\hline TNFR-1 & $2.6[2.1-4.1]$ & $3.1[2.3-4.2]$ & $3[2.4-5.2]$ & $5.1[4.1-8.6]$ & $<0.001$ \\
\hline Troy & $0.9[0.8-1.6]$ & $1[0.8-1.4]$ & $1.2[0.6-1.7]$ & $1.9[1.2-2.6]$ & $<0.001$ \\
\hline \multicolumn{6}{|l|}{ miR-199a-3p } \\
\hline Angiogenin & 3723.4 [2317.9-4907.5] & 3819.8 [3252.4-5453.6] & 4312.1 [2997.5-6143.4] & 4595.5 [3104.5-6748.6] & 0.024 \\
\hline Galectin-3 & $21.7 \pm 8.4$ & $21.8 \pm 8.8$ & $24.8 \pm 11.1$ & $27.3 \pm 10.7$ & 0.018 \\
\hline Neuropilin-1 & $871.8 \pm 221.1$ & $987 \pm 330.6$ & $1002.9 \pm 260.9$ & $1062.8 \pm 350.3$ & 0.018 \\
\hline \multicolumn{6}{|l|}{ miR-223-3p } \\
\hline Galectin-3 & $19.8 \pm 8.9$ & $23.4 \pm 9.3$ & $24 \pm 10.1$ & $28.4 \pm 10$ & 0.001 \\
\hline GDF-15 & $2.8[1.8-4]$ & $3.1[1.9-5.4]$ & $3.4[1.8-6.1]$ & $3.7[2.6-6.1]$ & 0.016 \\
\hline LTBR & $0.7[0.5-1.1]$ & $0.7[0.5-1.3]$ & $0.8[0.5-1.3]$ & $1[0.7-1.6]$ & 0.002 \\
\hline PIGR & 593.3 [440-1040.3] & 738.8 [420.9-1092.3] & 695.6 [408-904.3] & 976.3 [659.6-1367.6] & 0.004 \\
\hline RAGE & $3.1[2.1-4.2]$ & $3.6[2.1-5.8]$ & $3[2.2-5]$ & 5.7 [3.9-7.7] & 0.006 \\
\hline Syndecan-1 & 19 [16.6-24.7] & $23.5[15.1-28.3]$ & $20.9[16.2-30]$ & $22.8[20.3-34.2]$ & 0.023 \\
\hline TNFR-1 & $2.7[2.1-3.7]$ & $3.8[2.3-5.3]$ & $3.2[2.6-5.3]$ & $4.9[3.2-7.5]$ & 0.005 \\
\hline Troy & $1[0.8-1.6]$ & $1.2[0.8-1.7]$ & $1[0.7-1.6]$ & $1.5[1-2.5]$ & 0.001 \\
\hline VEGFR-1 & $0.9[0.1-1]$ & $0.9[0.6-1.2]$ & $0.9[0.6-1.2]$ & $0.7[0.5-1.7]$ & 0.029 \\
\hline \multicolumn{6}{|l|}{ miR-652-3p } \\
\hline RAGE & $3[2.3-4.5]$ & $3.3[2.1-5]$ & $3.9[2.1-6.2]$ & $5[3.1-7.5]$ & 0.024 \\
\hline
\end{tabular}

Biomarker values are presented in $\mathrm{ng} / \mathrm{mL}$ per quartile of circulating miRNA levels, either as mean \pm standard deviation or median with interquartile range (in square brackets). Quartile 1 (Q1) represents the patients with the highest miRNA levels, whereas quartile 4 (Q4) represents the patients with the lowest miRNA levels

$E S A M$ endothelial cell-selective adhesion molecule, GDF-15 growth differentiation factor 15, $L T B R$ lymphotoxin beta receptor, $N G A L$ neutrophil gelatinase-associated lipocalin, PIGR polymeric immunoglobulin receptor, RAGE receptor for advanced glycation endproducts, TNFR- 1 tumor necrosis factor alpha receptor 1, troy tumor necrosis factor receptor superfamily member, VEGFR-1 vascular endothelial growth receptor 1 


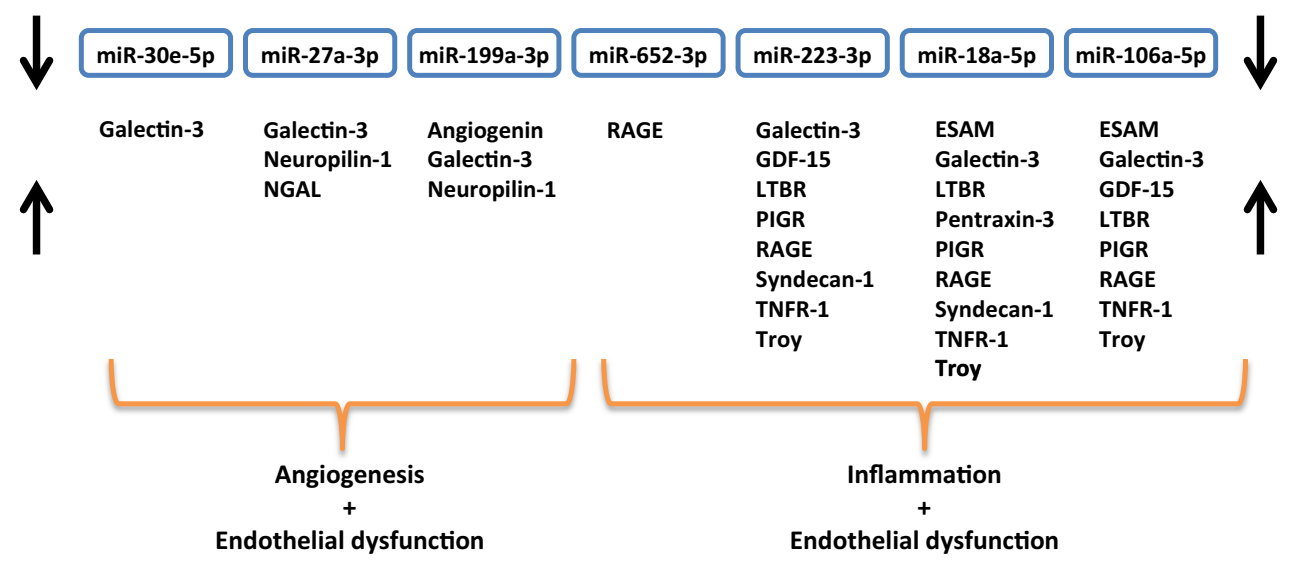

Fig. 1 Overview of the biomarker profile corresponding to low circulating microRNA levels. The depicted miRNAs are all lower expressed in plasma of heart failure patients with PAD and multiple manifestations of atherosclerotic disease. Low levels of these miRNAs are associated with high plasma levels of several biomarkers which are related to processes involved in atherosclerosis. ESAM endothelial cell-selective adhesion molecule, $G D F-15$ growth differentiation factor $15, L T B R$ lymphotoxin beta receptor, $N G A L$ neutrophil gelatinase-associated lipocalin, PIGR polymeric immunoglobulin receptor, $R A G E$ receptor for advanced glycation end products, TNFR1 tumor necrosis factor alpha receptor 1 and troy; tumor necrosis factor receptor superfamily member
$\mathrm{CV}$ causes (with exclusion of heart failure), of which $18(64 \%)$ were due to an atherosclerosis-related event (Supplementary Table 4). Univariable Cox proportional hazards analyses identified miR-106a-5p, miR-223-3p, miR-27a-3p, miR-16-5p, miR-30e-5p and let-7i-5p as significantly predictive for a $\mathrm{CV}$-related rehospitalization (Fig. 2), showing consistent associations of low miRNA

$\begin{array}{lrrr}\text { MicroRNA } & \text { HR } & \text { C-index } & \text { P-value } \\ \text { miR-18a-5p } & 1.353 & 0.566 & 0.097 \\ \text { miR-106a-5p } & 1.673 & 0.645 & 0.004 \\ \text { + age, sex, eGFR, log(BNP) } & 1.694 & 0.690 & 0.012 \\ \text { miR-26b-5p } & 1.217 & 0.558 & 0.343 \\ \text { miR-223-3p } & 1.460 & 0.625 & 0.020 \\ \text { + age, sex, eGFR, log(BNP) } & 1.478 & 0.660 & 0.039 \\ \text { miR-199a-3p } & 1.248 & 0.550 & 0.232 \\ \text { miR-27a-3p } & 1.437 & 0.592 & 0.034 \\ \text { + age, sex, eGFR, log(BNP) } & 1.482 & 0.650 & 0.027 \\ \text { miR-652-3p } & 1.415 & 0.595 & 0.059 \\ \text { miR-423-5p } & 1.114 & 0.522 & 0.558 \\ \text { miR-16-5p } & 1.839 & 0.650 & 0.002 \\ + \text { + age, sex, eGFR, log(BNP) } & 1.763 & 0.693 & 0.005 \\ \text { miR-30e-5p } & 1.475 & 0.606 & 0.044 \\ \text { + age, sex, eGFR, log(BNP) } & 1.432 & 0.665 & 0.079 \\ \text { let-7i-5p } & 1.793 & 0.632 & 0.005 \\ \text { + age, sex, eGFR, log(BNP) } & 2.058 & 0.700 & 0.002\end{array}$

Fig. 2 Predictive value of circulating microRNAs for cardiovascularrelated rehospitalizations within 18 months. Univariable Cox proportional hazards regression analyses were performed for all circulating miRNAs. Only univariable significant miRNAs $(P<0.05)$ were added to a clinical model including age, sex, eGFR and $\log (\mathrm{BNP})$. This levels with an increased risk of reaching the endpoint. The addition of clinically relevant variables including age, sex, b-type natriuretic peptide (BNP) and estimated glomerular filtration rate (eGFR) resulted in 5 miRNAs remaining significantly predictive. C-statistics identified the model with let-7i-5p as best performing with a C-index of 0.70 . The same analyses with these miRNAs

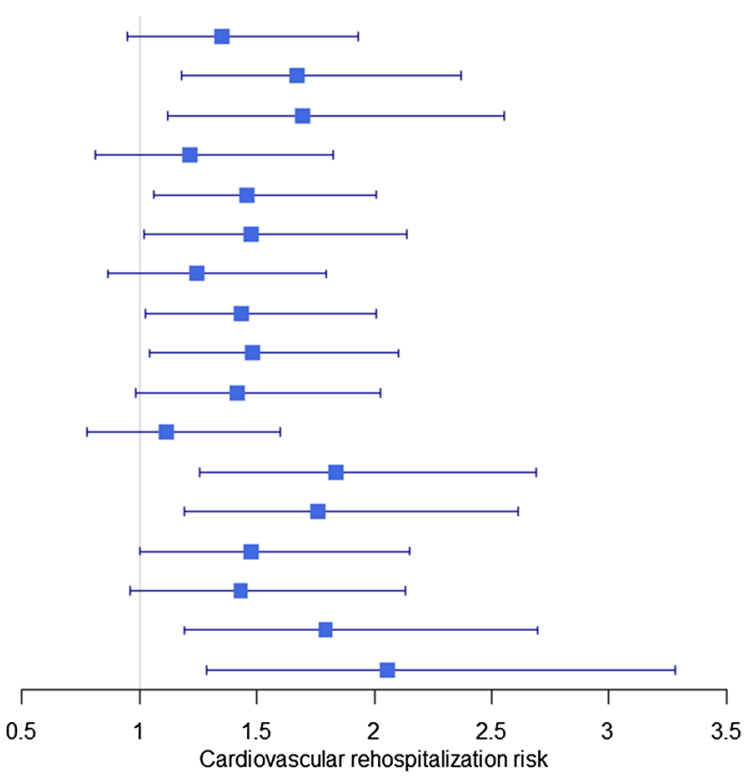

clinical model reached a C-index of 0.611 (all variables $P>0.05$ ). The hazard ratio (HR) is depicted with $95 \%$ confidence interval and should be interpreted per standard deviation. C-statistics were performed to assess model performance (presented as C-index) 
and other clinical endpoints including heart failure rehospitalization and mortality did not result in similar findings. No significant associations were identified for any of the miRNAs with all-cause mortality within 18 months and only miR-106a-5p was univariable predictive for a heart failure rehospitalization and the primary combined endpoint (heart failure rehospitalization and/ or death within 18 months), as presented in Supplementary Tables 5A-C. However, this association did not remain significant after adjustment for clinically relevant parameters.

\section{Discussion}

In the present study, we confirmed our previous finding of a specific set of miRNAs that were lower expressed in patients with heart failure compared with age-matched controls. Within our group of heart failure patients, several of these heart failure-related miRNAs were lower in patients with multiple manifestations of atherosclerotic disease, and PAD in particular. These results were supported by the finding that low levels of these miRNAs were associated with high levels of several biochemical markers related to inflammation, angiogenesis and endothelial dysfunction, which are all involved in the development and progression of atherosclerosis. Finally, low levels of six of these heart failure specific miRNAs were shown to predict the risk of a CV-related readmission after a heart failure hospitalization. These findings suggest a potential involvement of these miRNAs in atherosclerosis and related disease mechanisms.

\section{Circulating microRNAs and peripheral arterial disease}

Non-coronary atherosclerotic disease is a common comorbidity in heart failure patients and it has been shown to be an important predictor of the presence of CAD [23]. PAD in specific can be regarded to as generalized manifestation of atherosclerotic disease, which might explain why we found the most striking association between low levels of miRNAs and the presence of PAD. Few studies investigated the circulating miRNA profile in patients with PAD. Stather et al. [24] identified several downregulated circulating miRNAs related to PAD with similarities to our investigated circulating miRNA panel, including miR16 , miR-26b and miR-27b. Another study in patients with atherosclerotic abdominal aortic aneurysms found significantly upregulated miR-223 levels in atherosclerotic tissue, whereas miR-223 levels in plasma were downregulated [25], in concordance with our study.

\section{Associations between microRNAs} and atherosclerosis-related disease mechanisms

The potential involvement of these miRNAs in atherosclerosis-related processes was further supported by the association between low levels of circulating miRNAs and elevated levels of biomarkers related to inflammation, angiogenesis and endothelial dysfunction. Interestingly, these processes are all well-described disease mechanisms in both atherosclerosis $[16,26]$ and heart failure $[27,28]$.

Especially miR-18a-5p, miR-106a-5p and miR-223-3p were associated with a high number of mainly inflammatory and endothelium-related biomarkers, including ESAM, RAGE and pentraxin-3. Various roles for these biomarkers have been described, including migration of neutrophils and macrophages [29], leukocyte adhesion [30], endothelial dysfunction and vascular homeostasis [31]. The associations between these biomarkers and several heart failure-related miRNAs coincide with previous associations of miR-18a-5p, miR-106a-5p and miR-223-3p with inflammation and endothelial-related processes. In endothelial cells, miR-18a (part of the miR-17 92 cluster) was mainly described as anti-angiogenic [32], although a recent study reported that this cluster was required for endothelial cell proliferation and angiogenic sprouting after VEGF stimulation [10]. This suggests that the miR-17 92 cluster exhibits complex roles in endothelial cell function and angiogenesis, although the precise understanding of the underlying mechanisms warrants further investigation. MiR-223 is a well-known inflammation-related miRNA and is abundant in platelets, leukocytes and endothelial-derived microvesicles [11]. Besides its anti-angiogenic properties it was shown that miR-223 can function as potential contributor to the quiescence of endothelial cells [12]. Furthermore, MiR-106a has been associated to macrophage activation, suggesting involvement in inflammation [13].

We showed that low levels of miR-27a-3p and miR$199 a-3 p$ were associated with angiogenesis-related markers including angiogenin, neuropilin-1 and galectin-3. MiR-27a is present in endothelial cells and was previously described as key regulator of endothelial cell sprouting and angiogenesis [14], suggesting a substantial involvement in vascular dysfunction. MiR-199a-3p is mainly described as hypoxiarelated miRNA and can function as promoter of metastasis and angiogenesis [15]. A potential role for miR-199a-3p in angiogenesis is also reflected in the present study by the observed association with angiogenesis-related markers.

Our target prediction and network analyses also imply involvement of the investigated miRNAs in atherosclerosis-related processes, since targets as FOXO1 and CDK2 were previously shown to have key roles in the development of atherosclerosis, including angiogenesis, oxidative stress and proliferation of smooth muscle cells [33, 34]. 
Interestingly, both FOXO1 and MAPK14-another important node in our network-were also implicated in the development of heart failure [35, 36], therefore the identified targets may reflect key regulating mechanisms in both atherosclerotic disease and heart failure.

\section{Relation of circulating microRNAs to rehospitalization due to cardiovascular causes}

Besides the associations with the clinical phenotype and biochemical profile of atherosclerosis, we found very consistent associations between low levels of several miRNAs and CV-related rehospitalizations within 18 months, while no relations with other clinical endpoints including heart failure rehospitalization and mortality were found. Interestingly, most of the CV readmissions were related to atherosclerosis, suggesting that these miRNAs are able to predict the risk of atherosclerosis-related rehospitalizations in patients with heart failure.

Hospital readmission after a hospitalization for acute heart failure is a major problem and although biomarkers can predict response to acute heart failure treatment [37], few valuable predictors of long-term outcome besides the natriuretic peptides have been proposed so far [38]. Moreover, studies investigating the predictive value of circulating miRNAs in (acute) heart failure patients in relation to adverse outcome are scarce. Two studies identified miR423-5p as prognostic biomarker for a hospital readmission [39] and all-cause mortality [7] in acute heart failure patients, but in the current study this miRNA did not predict a CV-related rehospitalization. Here, we identified let7i-5p as strongest predictor of CV-related rehospitalizations and although there is no literature specifically addressing the relation of let-7i-5p with clinical outcome, the let-7 family has been described before in relation to $\mathrm{CV}$ disease [40]. Not all miRNAs with significant predictive value for $\mathrm{CV}$ rehospitalization overlap with the miRNAs found to be related to the atherosclerotic phenotype and vice versa, which may indicate that some miRNAs mainly reflect processes underlying atherosclerosis while others have a stronger association with progressing disease and outcome parameters. Nevertheless, we found a highly consistent pattern of lower miRNA levels associated with the atherosclerotic disease phenotype as well as an increased risk of $\mathrm{CV}$ rehospitalizations.

\section{Low circulating microRNA levels; increased uptake or decreased secretion?}

The consistent pattern of decreased circulating miRNA levels associated with different aspects of atherosclerotic disease is intriguing and leads to questions regarding their biological role in the circulation. One possible explanation for these low miRNA levels might lie in the increased uptake by recipient cells. It has been shown that circulating miRNAs can function in cell-to-cell communication and that recipient cells can engulf vesicle encapsulated miRNAs which consequently alters important cell functions [1]. In atherosclerosis, Zernecke et al. demonstrated in vitro that miR-126-enriched apoptotic bodies produced by endothelial cells can be taken up by vascular cells to regulate VEGF [41]. On the other hand, a diminished release of miRNAenriched vesicles could also lead to downregulated miRNA levels in plasma. Since increased angiogenesis is associated with plaque progression and instability in atherosclerosis, it has been speculated that a reduced export of angiogenic miRNAs outside cells might inhibit pro-angiogenic signaling [42]. Indeed, in serum of patients with CAD, it has been shown that extracellular vesicles are loaded with less CAD-related miRNAs in comparison to healthy subjects [43]. However, the majority of the extracellular miRNAs are vesicle-free and bound to Ago proteins, of which no evidence of miRNA trafficking is currently available. Therefore, further research is needed to unravel the precise underlying mechanisms of reduced circulating miRNA levels in patients with heart failure and atherosclerosis.

\section{Study limitations}

The limitations of this study should be acknowledged. First, although very consistent miRNA patterns were found, the studied patient population was relatively small. Second, the associations of heart failure-related circulating miRNAs with atherosclerosis and their role in common disease mechanisms such as vascular dysfunction should be further investigated in experimental settings to determine causal links.

\section{Conclusions}

Although the precise functions of circulating miRNAs in heart failure are still elusive, this study proposes a link between downregulated heart failure-related miRNAs and the presence of atherosclerosis and provides insight into potential related pathophysiological mechanisms including angiogenesis, inflammation and endothelial dysfunction. Further, we show the predictive value of these circulating miRNAs for the risk of a CV rehospitalization in heart failure patients. Future studies may elucidate the involvement of circulating miRNAs in heart failure and atherosclerosisrelated disease pathways, potentially leading to novel biomarkers and drug targets.

Acknowledgements We acknowledge the support from the Netherlands CardioVascular Research Initiative: the Netherlands Heart 
Foundation, Dutch Federation of University Medical Centres, the Netherlands Organization for Health Research and Development and the Royal Netherlands Academy of Sciences. This study was further supported by a Grant from the Netherlands Heart Foundation: Approaching Heart Failure By Translational Research Of RNA Mechanisms (ARENA, CVON-2011-11). The COACH trial was supported by Grant $2000 Z 003$ from the Netherlands Heart Foundation and by additional unrestricted Grants from Biosite France SAS, Jouy-en-Josas, France (brain natriuretic peptide), Roche Diagnostics Nederland BV, Venlo, the Netherlands (N-terminal prohormone brain natriuretic peptide), and Novartis Pharma BV, Arnhem, the Netherlands.

\section{Compliance with ethical standards}

Ethical standards The $\mathrm{COACH}$ and Telosophy study were approved by the appropriate ethics committees and were performed in accordance with the ethical standards laid down in the 1964 Declaration of Helsinki and its later amendments. All patients provided written informed consent

Conflict of interest D. J. V. V., E. L. V. and T. J. have nothing to disclose. E. B. is a co-founder and member of the scientific advisory board of InteRNA Technologies B. V., which develops miRNA therapeutics for cancer. A. A. V., E. B., E. S. O. and P. V. D. M. are patent holders of the circulating miRNAs in this manuscript. No other conflicts were reported.

Open Access This article is distributed under the terms of the Creative Commons Attribution 4.0 International License (http:// creativecommons.org/licenses/by/4.0/), which permits unrestricted use, distribution, and reproduction in any medium, provided you give appropriate credit to the original author(s) and the source, provide a link to the Creative Commons license, and indicate if changes were made.

\section{References}

1. Vegter EL, van der Meer P, de Windt LJ, Pinto YM, Voors AA (2016) MicroRNAs in heart failure: from biomarker to target for therapy. Eur J Heart Fail 18:457-468

2. Small EM, Olson EN (2011) Pervasive roles of microRNAs in cardiovascular biology. Nature 469:336-342

3. Bruno N, Ter Maaten JM, Ovchinnikova ES, Vegter EL, Valente MA, van der Meer P, de Boer RA, van der Harst P, Schmitter D, Metra M, O'Connor CM, Ponikowski P, Teerlink JR, Cotter G, Davison B, Cleland JG, Givertz MM, Bloomfield DM, Dittrich HC, Pinto YM, van Veldhuisen DJ, Hillege HL, Berezikov E, Voors AA (2016) MicroRNAs relate to early worsening of renal function in patients with acute heart failure. Int J Cardiol 203:564-569

4. Vegter EL, Schmitter D, Hagemeijer Y, Ovchinnikova ES, van der Harst P, Teerlink JR, O'Connor CM, Metra M, Davison BA, Bloomfield D, Cotter G, Cleland JG, Givertz MM, Ponikowski $\mathrm{P}$, van Veldhuisen DJ, van der Meer P, Berezikov E, Voors AA, Khan MA (2016) Use of biomarkers to establish potential role and function of circulating microRNAs in acute heart failure. Int J Cardiol 224:231-239

5. Ellis KL, Cameron VA, Troughton RW, Frampton CM, Ellmers LJ, Richards AM (2013) Circulating microRNAs as candidate markers to distinguish heart failure in breathless patients. Eur J Heart Fail 15:1138-1147
6. Goren Y, Kushnir M, Zafrir B, Tabak S, Lewis BS, Amir O (2012) Serum levels of microRNAs in patients with heart failure. Eur J Heart Fail 14:147-154

7. Ovchinnikova ES, Schmitter D, Vegter EL, Ter Maaten JM, Valente MA, Liu LC, van der Harst P, Pinto YM, de Boer RA, Meyer S, Teerlink JR, O'Connor CM, Metra M, Davison BA, Bloomfield DM, Cotter G, Cleland JG, Mebazaa A, Laribi S, Givertz MM, Ponikowski P, van der Meer P, van Veldhuisen DJ, Voors AA, Berezikov E (2016) Signature of circulating microRNAs in patients with acute heart failure. Eur J Heart Fail 18:414-423

8. Zampetaki A, Willeit P, Tilling L, Drozdov I, Prokopi M, Renard JM, Mayr A, Weger S, Schett G, Shah A, Boulanger CM, Willeit J, Chowienczyk PJ, Kiechl S, Mayr M (2012) Prospective study on circulating microRNAs and risk of myocardial infarction. J Am Coll Cardiol 60:290-299

9. Akat KM, Moore-McGriff D, Morozov P, Brown M, Gogakos T, Correa Da Rosa J, Mihailovic A, Sauer M, Ji R, Ramarathnam A, Totary-Jain H, Williams Z, Tuschl T, Schulze PC (2014) Comparative RNA-sequencing analysis of myocardial and circulating small RNAs in human heart failure and their utility as biomarkers. Proc Natl Acad Sci USA 111(30):11151-11156

10. Chamorro-Jorganes A, Lee MY, Araldi E, Landskroner-Eiger S, Fernandez-Fuertes M, Sahraei M, Quiles Del Rey M, van Solingen C, Yu J, Fernandez-Hernando C, Sessa WC, Suarez $\mathrm{Y}$ (2016) VEGF-induced expression of miR-17-92 cluster in endothelial cells is mediated by ERK/ELK1 activation and regulates angiogenesis. Circ Res 118:38-47

11. Shan Z, Qin S, Li W, Wu W, Yang J, Chu M, Li X, Huo Y, Schaer GL, Wang S, Zhang C (2015) An endocrine genetic signal between blood cells and vascular smooth muscle cells: role of microRNA-223 in smooth muscle function and atherogenesis. J Am Coll Cardiol 65:2526-2537

12. Shi L, Fisslthaler B, Zippel N, Fromel T, Hu J, Elgheznawy A, Heide H, Popp R, Fleming I (2013) MicroRNA-223 antagonizes angiogenesis by targeting beta1 integrin and preventing growth factor signaling in endothelial cells. Circ Res 113:1320-1330

13. Zhu D, Pan C, Li L, Bian Z, Lv Z, Shi L, Zhang J, Li D, Gu H, Zhang CY, Liu Y, Zen K (2013) MicroRNA-17/20a/106a modulate macrophage inflammatory responses through targeting signal-regulatory protein alpha. J Allergy Clin Immunol 132(426-36):e8

14. Urbich C, Kaluza D, Fromel T, Knau A, Bennewitz K, Boon RA, Bonauer A, Doebele C, Boeckel JN, Hergenreider E, Zeiher AM, Kroll J, Fleming I, Dimmeler S (2012) MicroRNA-27a/b controls endothelial cell repulsion and angiogenesis by targeting semaphorin 6A. Blood 119:1607-1616

15. Pencheva N, Tran H, Buss C, Huh D, Drobnjak M, Busam K, Tavazoie SF (2012) Convergent multi-miRNA targeting of ApoE drives LRP1/LRP8-dependent melanoma metastasis and angiogenesis. Cell 151:1068-1082

16. Faxon DP, Fuster V, Libby P, Beckman JA, Hiatt WR, Thompson RW, Topper JN, Annex BH, Rundback JH, Fabunmi RP, Robertson RM, Loscalzo J, American Heart Association (2004) Atherosclerotic vascular disease conference: writing group III: pathophysiology. Circulation 109:2617-2625

17. Jaarsma T, van der Wal MH, Lesman-Leegte I, Luttik ML, Hogenhuis J, Veeger NJ, Sanderman R, Hoes AW, van Gilst WH, Lok DJ, Dunselman PH, Tijssen JG, Hillege HL, van Veldhuisen DJ, Coordinating Study Evaluating Outcomes of Advising and Counseling in Heart Failure (COACH) Investigators (2008) Effect of moderate or intensive disease management program on outcome in patients with heart failure: coordinating study evaluating outcomes of advising and counseling in heart failure (COACH). Arch Intern Med 168:316-324 
18. Wong LS, Huzen J, de Boer RA, van Gilst WH, van Veldhuisen DJ, van der Harst P (2011) Telomere length of circulating leukocyte subpopulations and buccal cells in patients with ischemic heart failure and their offspring. PLoS One 6:e23118

19. Christenson RH, Duh SH, Wu AH, Smith A, Abel G, deFilippi CR, Wang S, Adourian A, Adiletto C, Gardiner P (2010) Multicenter determination of galectin-3 assay performance characteristics: anatomy of a novel assay for use in heart failure. Clin Biochem 43:683-690

20. Meijers WC, de Boer RA, van Veldhuisen DJ, Jaarsma T, Hillege HL, Maisel AS, Di Somma S, Voors AA, Peacock WF (2015) Biomarkers and low risk in heart failure. Data from $\mathrm{COACH}$ and TRIUMPH. Eur J Heart Fail 17:1271-1282

21. Chou CH, Chang NW, Shrestha S, Hsu SD, Lin YL, Lee WH, Yang CD, Hong HC, Wei TY, Tu SJ, Tsai TR, Ho SY, Jian TY, Wu HY, Chen PR, Lin NC, Huang HT, Yang TL, Pai CY, Tai CS, Chen WL, Huang CY, Liu CC, Weng SL, Liao KW, Hsu WL, Huang HD (2016) miRTarBase 2016: updates to the experimentally validated miRNA-target interactions database. Nucleic Acids Res 44:D239-D247

22. Szklarczyk D, Franceschini A, Wyder S, Forslund K, Heller D, Huerta-Cepas J, Simonovic M, Roth A, Santos A, Tsafou KP, Kuhn M, Bork P, Jensen LJ, von Mering C (2015) STRING v10: protein-protein interaction networks, integrated over the tree of life. Nucleic Acids Res 43:D447-D452

23. Weissgerber A, Scholz M, Teren A, Sandri M, Teupser D, Gielen S, Thiery J, Schuler G, Beutner F (2016) The value of noncoronary atherosclerosis for identifying coronary artery disease: results of the Leipzig LIFE Heart Study. Clin Res Cardiol 105:172-181

24. Stather PW, Sylvius N, Wild JB, Choke E, Sayers RD, Bown MJ (2013) Differential microRNA expression profiles in peripheral arterial disease. Circ Cardiovasc Genet 6:490-497

25. Kin K, Miyagawa S, Fukushima S, Shirakawa Y, Torikai K, Shimamura K, Daimon T, Kawahara Y, Kuratani T, Sawa Y (2012) Tissue- and plasma-specific MicroRNA signatures for atherosclerotic abdominal aortic aneurysm. J Am Heart Assoc 1:e000745

26. Dopheide JF, Geissler P, Rubrech J, Trumpp A, Zeller GC, Daiber A, Munzel T, Radsak MP, Espinola-Klein C (2016) Influence of exercise training on proangiogenic TIE-2 monocytes and circulating angiogenic cells in patients with peripheral arterial disease. Clin Res Cardiol 105:666-676

27. Colombo PC, Onat D, Harxhi A, Demmer RT, Hayashi Y, Jelic S, LeJemtel TH, Bucciarelli L, Kebschull M, Papapanou P, Uriel N, Schmidt AM, Sabbah HN, Jorde UP (2014) Peripheral venous congestion causes inflammation, neurohormonal, and endothelial cell activation. Eur Heart J 35:448-454

28. Poss J, Ukena C, Kindermann I, Ehrlich P, Fuernau G, Ewen S, Mahfoud F, Kriechbaum S, Bohm M, Link A (2015) Angiopoietin-2 and outcome in patients with acute decompensated heart failure. Clin Res Cardiol 104:380-387

29. Inoue M, Ishida T, Yasuda T, Toh R, Hara T, Cangara HM, Rikitake Y, Taira K, Sun L, Kundu RK, Quertermous T, Hirata K (2010) Endothelial cell-selective adhesion molecule modulates atherosclerosis through plaque angiogenesis and monocyteendothelial interaction. Microvasc Res 80:179-187

30. Kierdorf K, Fritz G (2013) RAGE regulation and signaling in inflammation and beyond. J Leukoc Biol 94:55-68
31. Carrizzo A, Lenzi P, Procaccini C, Damato A, Biagioni F, Ambrosio M, Amodio G, Remondelli P, Del Giudice C, Izzo R, Malovini A, Formisano L, Gigantino V, Madonna M, Puca AA, Trimarco B, Matarese G, Fornai F, Vecchione C (2015) Pentraxin 3 induces vascular endothelial dysfunction through a P-selectin/matrix metalloproteinase-1 pathway. Circulation 131:1495-1505

32. Doebele C, Bonauer A, Fischer A, Scholz A, Reiss Y, Urbich C, Hofmann WK, Zeiher AM, Dimmeler S (2010) Members of the microRNA-17-92 cluster exhibit a cell-intrinsic antiangiogenic function in endothelial cells. Blood 115:4944-4950

33. Lange M, Fujikawa T, Koulova A, Kang S, Griffin MJ, Lassaletta AD, Erat A, Tobiasch E, Bianchi C, Elmadhun N, Sellke FW, Usheva A (2014) Arterial territory-specific phosphorylated retinoblastoma protein species and CDK2 promote differences in the vascular smooth muscle cell response to mitogens. Cell Cycle 13:315-323

34. Papanicolaou KN, Izumiya Y, Walsh K (2008) Forkhead transcription factors and cardiovascular biology. Circ Res 102:16-31

35. Qi Y, Xu Z, Zhu Q, Thomas C, Kumar R, Feng H, Dostal DE, White MF, Baker KM, Guo S (2013) Myocardial loss of IRS1 and IRS2 causes heart failure and is controlled by p38alpha MAPK during insulin resistance. Diabetes 62:3887-3900

36. Yokota T, Wang Y (2016) p38 MAP kinases in the heart. Gene 575:369-376

37. ter Maaten JM, Valente MA, Metra M, Bruno N, O'Connor CM, Ponikowski P, Teerlink JR, Cotter G, Davison B, Cleland JG, Givertz MM, Bloomfield DM, Dittrich HC, van Veldhuisen DJ, Hillege HL, Damman K, Voors AA (2016) A combined clinical and biomarker approach to predict diuretic response in acute heart failure. Clin Res Cardiol 105:145-153

38. Frioes F, Lourenco P, Laszczynska O, Almeida PB, Guimaraes JT, Januzzi JL, Azevedo A, Bettencourt P (2015) Prognostic value of sST2 added to BNP in acute heart failure with preserved or reduced ejection fraction. Clin Res Cardiol 104:491-499

39. Seronde MF, Vausort M, Gayat E, Goretti E, Ng LL, Squire IB, Vodovar N, Sadoune M, Samuel JL, Thum T, Solal AC, Laribi S, Plaisance P, Wagner DR, Mebazaa A, Devaux Y, GREAT network (2015) Circulating microRNAs and outcome in patients with acute heart failure. PLoS One 10:e0142237

40. Bao MH, Feng X, Zhang YW, Lou XY, Cheng Y, Zhou HH (2013) Let-7 in cardiovascular diseases, heart development and cardiovascular differentiation from stem cells. Int J Mol Sci 14:23086-23102

41. Zernecke A, Bidzhekov K, Noels H, Shagdarsuren E, Gan L, Denecke B, Hristov M, Koppel T, Jahantigh MN, Lutgens E, Wang S, Olson EN, Schober A, Weber C (2009) Delivery of microRNA-126 by apoptotic bodies induces CXCL12-dependent vascular protection. Sci Signal 2:ra81

42. Finn NA, Searles CD (2012) Intracellular and Extracellular miRNAs in Regulation of Angiogenesis Signaling. Curr Angiogenes 4:299-307

43. Finn NA, Eapen D, Manocha P, Al Kassem H, Lassegue B, Ghasemzadeh N, Quyyumi A, Searles CD (2013) Coronary heart disease alters intercellular communication by modifying microparticle-mediated microRNA transport. FEBS Lett 587:3456-3463 